\title{
Miocardiopatía por Influenza A H1N1. Reporte de un caso clínico
}

\author{
Influenza A H1N1 Cardiomyopathy. Report of a clinical case \\ Cardiomiopatia por Influenza A H1N1. Relato de um caso clínico
}

Sofía Rostán Resumen: La miocarditis es una enfermedad inflamatoria del miocardio secundaria a enfermedades

ORCID: 0000-0003-2206-5684 Residente Medicina Interna.

Natasha Smiliansky

ORCID: 0000-0002-8254-5682 Residente Medicina Interna.

Andrea Vaucher ORCID: 0000-0002-5574-7596 Internista. Profesora Adjunta Clínica Médica. autoinmunes, tóxicas e infecciosas. Dentro de éstas últimas, las virales son las más frecuentes. Sin embargo, el virus de influenza A H1N1 sigue siendo una etiología poco reportada. La prevalencia de miocarditis fulminante debido a gripe estacional ha sido establecida entre 1 y $11 \%$ según los criterios diagnósticos utilizados, y la prevalencia de miocarditis causada por H1N1 se estima en 13\%. Presentamos un caso clínico, de una paciente joven, donde se realiza diagnóstico retrospectivo de Miocarditis por Influenza A H1N1 con presentación de miocarditis fulminante, con alteraciones electrocardiográficas sugestivas, troponinas cardiacas positivas, caída de la función ventricular con posterior recuperación, y aislamiento microbiológico de agente causal.

Palabras claves: miocarditis, Influenza A H1N1

Abstract: Myocarditis is an inflammatory myocardial disease secondary to autoimmune, toxic, and infectious diseases. Among the latter, virals are the most frequent. However, influenza A $\mathrm{H} 1 \mathrm{~N} 1$ virus remains a poorly reported etiology. The prevalence of fulminant myocarditis due to seasonal influenza has been established between 1 and $11 \%$ according to the diagnostic criteria used, and the prevalence of myocarditis caused by $\mathrm{H} 1 \mathrm{~N} 1$ is estimated at $13 \%$. We report a clinical case, of a young patient, where a retrospective diagnosis of $\mathrm{H} 1 \mathrm{~N} 1$ Influenza A myocarditis was made with a presentation of fulminant myocarditis, with suggestive electrocardiographic abnormalities, positive cardiac troponins, loss of ventricular function with subsequent recovery, and microbiological isolation of the causal agent.

Key words: myocarditis, Influenza A H1N1

Resumo: A miocardite é uma doença inflamatória do miocárdio secundária a doenças autoimunes, tóxicas e infecciosas. Entre os últimos, os virais são os mais frequentes. No entanto, o vírus influenza A H1N1 permanece uma etiologia pouco relatada. A prevalência de miocardite fulminante por influenza sazonal foi estabelecida entre 1 e $11 \%$, de acordo com os critérios diagnósticos utilizados, e a prevalência de miocardite causada pelo H1N1 é estimada em 13\%. Apresentamos um caso clínico, de um paciente jovem, em que foi feito um diagnóstico retrospectivo de miocardite por influenza $\mathrm{A} \mathrm{H} 1 \mathrm{~N} 1$, com apresentação de miocardite fulminante, com alterações eletrocardiográficas sugestivas, troponinas cardíacas positivas, perda de função ventricular com recuperação subsequente e isolamento microbiológico do agente causal.

Palavras-chave: miocardite, Influenza A H1N1

Fecha recibido: 03/06/2020 Fecha aceptado: 12/10/2020

Clínica Médica 3. Prof. Dr. Alvaro Huarte. Hospital Maciel. Facultad de Medicina. Universidad de la República (UdelaR)

Montevideo, Uruguay.

Correspondencia: E-mail: sofiarostan@gmail.com 


\section{Introducción}

La miocarditis se define como una enfermedad inflamatoria del músculo cardiaco y es causa importante de miocardiopatía dilatada, insuficiencia cardiaca y muerte súbita en pacientes jóvenes. ${ }^{(1,2)}$ Dentro de las principales etiologías se encuentran las infecciones virales, siendo los agentes más frecuentemente involucrados los virus que tienen predilección por el miocardio: enterovirus, adenovirus, parvovirus B19, virus herpes humano tipo 6, Citomegalovirus, virus de Epstein Barr. ${ }^{(3)}$

La presentación clínica se caracteriza por un cuadro de impregnación viral, con fiebre, síntomas respiratorios y/o digestivos. Las manifestaciones cardiacas pueden variar en diferentes grados de falla cardiaca, desde insuficiencia cardiaca congestiva hasta shock cardiogénico con requerimiento de inotrópicos. Otras formas de presentación incluyen dolor torácico atípico, arritmias ventriculares y pericarditis. ${ }^{(4)}$

En la práctica médica son múltiples los estudios de laboratorio que pueden acercarnos al diagnóstico, los parámetros inflamatorios se encontrarán elevados, al igual que las troponinas cardiacas. En el electrocardiograma (ECG) se puede apreciar alteración de la onda T, del segmento ST, elevación del segmento ST que se asemeja a la imagen de infarto agudo de miocardio y trastornos de la conducción. También ensanchamiento del QRS el cual es considerado factor de riesgo independiente relacionado con la evolución y muerte súbita. La ecocardiografía continúa siendo fundamental para analizar la función ventricular en los pacientes con sospecha de miocarditis y es útil para descartar otras entidades diagnósticas. Sus hallazgos son inespecíficos ${ }^{(1,4)}$. Se debe realizar tanto en la presentación inicial como en el seguimiento del paciente. La resonancia magnética ha demostrado ser de gran utilidad para apoyar el diagnóstico de miocarditis. ${ }^{(1,2,5)}$. La biopsia miocárdica continúa siendo el patrón oro para el diagnóstico definitivo. ${ }^{(1,6)}$ En cuanto al agente causal, la infección viral se podrá confirmar por reacción en cadena polimerasa (PCR).

Actualmente, el tratamiento se encuentra dirigido hacia los síntomas de insuficiencia cardiaca y el soporte de la función sistólica, no existiendo tratamiento específico.

La mortalidad de la miocarditis aguda varía entre un $25 \%$ y un $50 \%$. Los pacientes que se presentan con falla cardiaca y con disfunción sistólica del ventrículo izquierdo, pueden tardar semanas o meses en recuperarse. En la mitad de los pacientes esta se recuperará completamente, mientras que el otro $50 \%$ desarrollará miocardiopatía dilatada con disfunción sistólica crónica. ${ }^{(7)}$

\section{Caso Clínico}

Se trata de una paciente de 51 años, sexo femenino. Antecedentes personales diabetes mellitus tipo 2 insulino requiriente (DMT2IR) con mal control metabólico. Cardiopatía isquémicahipertensiva. Trastorno depresivo en tratamiento con psicofármacos.

Consulta en emergencia del Hospital Maciel por cuadro de 7 días de evolución dado por fiebre, mialgias, artralgias, cefalea, rinorrea y tos seca, en la evolución agrega disnea a mínimos esfuerzos.

Del examen físico se destaca paciente lucida, apirética. A nivel cardiovascular ritmo regular de $90 \mathrm{cpm}$, sin elementos de falla cardiaca, a nivel pleuropulmonar murmullo alveovesicular presente, con estertores secos difusos. Saturación de oxígeno de $90 \%$ al aire.

Con planteo de infección respiratoria baja se realiza una radiografía de tórax (Figura 1). 


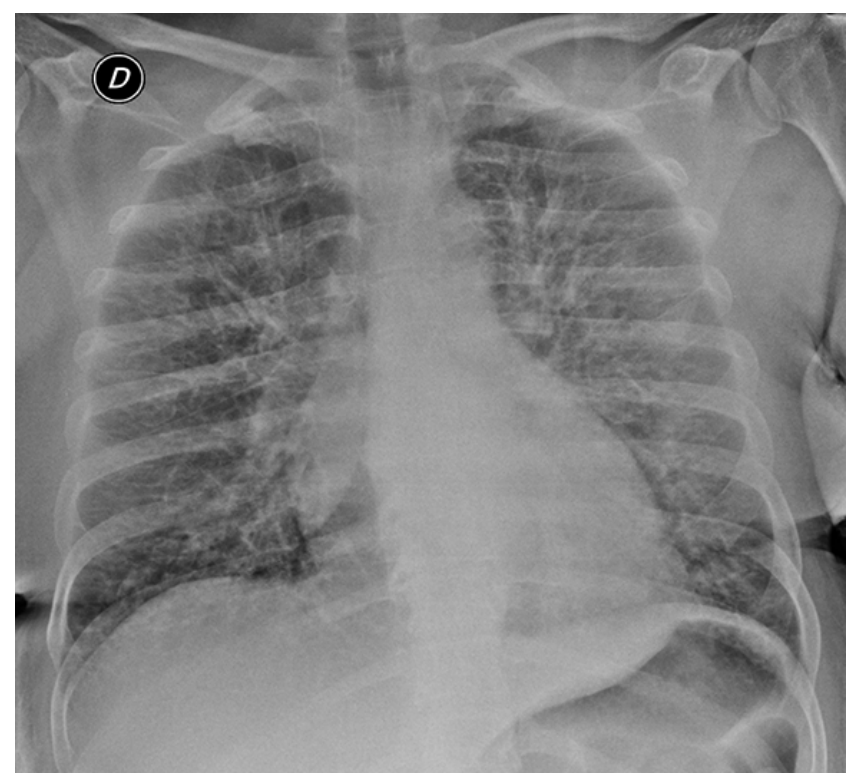

Tomografía de tórax compatible con neumonitis bilateral. Panel viral respiratorio positivo para Influenza A H1N1. La gasometría arterial mostraba una insuficiencia respiratoria tipo 1 leve.

Ingresa a sala de cuidados moderados bajo tratamiento con Oseltamivir. En la evolución presenta peoría de la insuficiencia respiratoria que requiere ingreso a Unidad de Cuidados Intensivos (UCl) y apoyo ventilatorio con ventilación no invasiva. Luego de 48 horas en UCl instala elementos de insuficiencia cardiaca congestiva, con repercusión hemodinámica, sin peoría del compromiso respiratorio, sin otras disfunciones.

Se realiza electrocardiograma (Figura 2), troponinas cardiacas de valor intermedias y ecocardiograma (ETT) que evidencia hipertrofia excéntrica del ventrículo izquierdo, alteraciones sectoriales, aquinesia septal e inferior en todos sus sectores, con una fracción de eyección del ventrículo izquierdo (FEVI) de 30\%. Aurícula izquierda moderada a severamente dilatada, insuficiencia aórtica y mitral leve. Función del ventrículo derecho conservada.
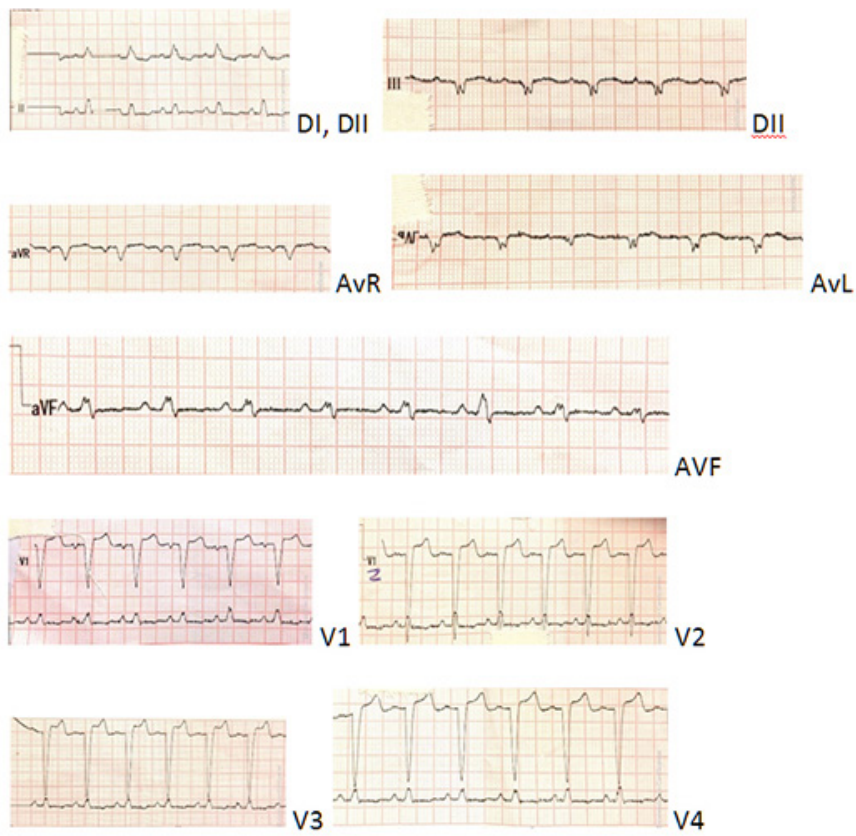

Figura 2: Electrocardiograma 12 derivaciones. Taquicardia sinusal y bloqueo completo de rama izquierda (BCRI) ya presente en electros previos
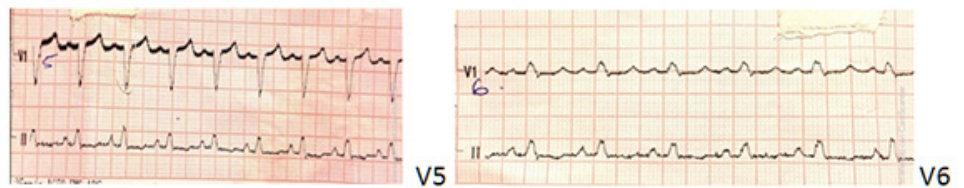
Se interpreta como compromiso miocárdico de infección respiratoria grave, se realiza tratamiento de soporte con inotrópicos y vasopresores. A las 48 horas de estabilidad, se traslada a sala de cuidados moderados. Al octavo día de tratamiento con oseltamivir, la paciente se encontraba sin insuficiencia respiratoria, sin insuficiencia cardiaca, sin fiebre. De la paraclínica se destacaba anemia moderada microcítica hipocrómica con hiperplaquetosis reactiva. Se solicita nuevo ETT que informa ventrículo izquierdo con leve aumento de su masa, FEVI normal, sin alteraciones de la motilidad segmentaria del VI. La paciente se fue de alta y continua en control en este centro.

Se realiza diagnóstico retrospectivo de Miocarditis por H1N1 con presentación de miocarditis fulminante. No contamos con resonancia nuclear magnética (RNM) debido a que el diagnóstico se realizó de forma retrospectivo. Pero apoyan el diagnóstico las alteraciones electrocardiográficas sugestivas, troponinas cardiacas positivas, caída de la función ventricular con posterior recuperación y aislamiento microbiológico de agente causal.

\section{Discusión}

Hemos presentado a una paciente joven con factores de riesgo cardiovasculares que se presenta con un síndrome febril acompañado de síntomas respiratorios, en la que se identifica el agente causal (Influenza A H1N1). En la evolución presenta una peoría clínica que se interpreta retrospectivamente como presentación de miocarditis fulminante causada por dicho microorganismo.

Debido a la pandemia de 2009 de gripe A H1N1 la presentación clínica de la enfermedad causada por la misma ha sido ampliamente descripta. Sin embargo, ha sido escasa la referencia al compromiso miocárdico de esta enfermedad. La prevalencia de miocarditis fulminante debido a gripe estacional ha sido establecida entre 1 y $11 \%$ según los criterios diagnósticos utilizados, y la prevalencia de miocarditis causada por $\mathrm{H} 1 \mathrm{~N} 1$ se estima en $13 \%^{(7,8)}$

De la literatura internacional se desprende que la edad de presentación se estima en una media de 40 años, y predomina en el sexo femenino. El inicio de los síntomas respiratorios es de 3-4 días previo a la presentación miocárdica, lo que manifiesta lo agudo de la enfermedad. Habitualmente se presenta a forma de miocarditis fulminante, que es aquella que requiere soporte mecánico circulatorio o altas dosis de catecolaminas, logrando una buena evolución posterior. ${ }^{(9,10,11)}$

El diagnóstico es mayoritariamente clínico, en cuanto a los estudios diagnósticos el inicial suele ser el ecocardiograma, y el único en la mayoría de los casos. Este evidencia en el $90 \%$ de los pacientes hipoquinesia difusa o focal con caída de la función ventricular siendo la promedio de $25 \%$, tal como se observó en el caso clínico. ${ }^{(5)}$ La RNM ha cobrado importancia y resulta ser más útil que la biopsia miocárdica, no solo por ser no invasiva, sino que además estima actividad y grado de inflamación miocárdica. La dificultad a la hora de su indicación radica en su baja disponibilidad. ${ }^{(5,12)}$

En cuanto al pronóstico se estima una recuperación de la función ventricular entre un 10 a $15 \%$ entre los días 4 y 22, tal como se observó en el caso reportado. ${ }^{(5,13,14)}$

\section{Conclusiones}

La gripe A H1N1 dada la situación epidemiológica actual debe tenerse en cuenta en el diagnóstico diferencial de la miocarditis viral. La instauración inmediata del tratamiento con oseltamivir puede modificar el curso clínico de la enfermedad, no existiendo actualmente otros tratamientos para la misma, además del tratamiento de soporte cardio- circulatorio.

\section{Bibliografía}

1- Dominguez F, Kühl U, Pieske B, Garcia-Pavia P, Tschöpe C. Update on Myocarditis and Inflammatory Cardiomyopathy: Reemergence of Endomyocardial Biopsy. Rev Esp Cardiol (Engl Ed). 2016 Feb;69(2):178-87. doi: 10.1016/j.rec.2015.10.015.

2- Abelleira C. Miocarditis. En: Sociedad Española de Cardiología Pediátrica y Cardiopatías Congénitas. Libro de protocolos [Internet]. Madrid: SECPCC, 2008 [Citado 06/09/2020]: 617-30. Disponible en: http://www.secardioped.org/Descargas/PyB/LP_cap43.pdf.

3- Kuhl U, Pauschinger M, Noutsias M, Seeberg B, BockT, Lassner D, et al. High prevalence of viral genomes and multiple viral infections in the myocardius of adults with "idiopathic" lefventricular dysfunction. Circulation. 2005; 111:887-893. 
4- Caforio AL, Pankuweit S, Arbustini E, Basso C, Gimeno-Blanes J, Felix SB, et al. Current state of knowledge on aetiology, diagnosis, management, and therapy of myocarditis: a position statement of the European Society of Cardiology Working Group on Myocardial and Pericardial Diseases. Eur Heart J. 2013; 34:v2636-2648.

5- Godínez-Baca LE, Luna-Pérez D. Miocarditis aguda por influenza AH1N1: reporte de caso. Rev Mex Cardiol. 2016;27(Suppl: 1):47-50.

6- Cooper LT, Baughman KL, Feldman AM, Frustaci A, Jessup M, Kuhl U, et al. The role of endomyocardial biopsy in the management of cardiovascular disease: A scientific statement from the American Heart Association, the American College of Cardiology, and the European Society of Cardiology Endorsed by the Heart Failure Society of America and the Heart Failure Association of the European Society of Cardiology. Eur Heart J. 2007;28: 3076-3093.

7- Pollack A, Kontorovich A, Fuster V, Dec G. Viral myocarditis diagnosis, treatment options, and current controversies. Nature Reviews Cardiology. 2015;12(11):670-680.

8- Gross ER, Reichstein A: Fulminant pH1N1-09 influenza-associated myocarditis in pediatric patients. Pediatr Crit Care Med. 2011 Mar; 12(2): e99-e101.

9- Shin SY, Kim JH, Kim HS. Clinical Characteristics of Korean Pediatric Patients Critically III With Influenza A (H1N1) Virus. Pediatr Pulmonol 2010 Jul 20.

10- Silva E, Montenegro JS, Estupiñan MC, Arias G, Osorio JP, Poveda CM, Buitrago R. Miocarditis fulminante en adultos por el virus Influenza B: reporte de casos y revisión de la literatura. Biomédica 2019: 39;(Supl2): 11-19.

11- Martín-Bermúdez R, Martínez-Roldán A, Jiménez-Jiménez J, Dusseck-Brutus R, Porras-López M, Pérez-Bernal J. Miocarditis fulminante asociada a influenza A. Med Intensiva. 2015;39(5):318-320.

12- Takeuchi I, Imaki R, Inomata T, Soma K, Izumi T. MRI is useful for diagnosis of H1N1 fulminant myocarditis. Circ J. 2010; 74 (12): 2758-2759.

13- Martin SS, Hollingworth CL, Norfolk SG, Wolfe CR, Hollingsworth JW. Reversible cardiac dysfunction associated with pandemic 2009 influenza A H1N1. Chest. 2010; 137 (5): 1195-1197.

14- Chotpitayasunondh T, Ungchusak K, Hanshaoworakul W, Chunsuthiwat S, Sawanpanyalert P, Lochindarat $S$ et al. Human disease from influenza A H5N1, Thailand, 2004. Emerg Infect Dis. 2005; 11(2): 201-209.

\section{Aporte cada autor al trabajo}

Sofía Rostán: concepción y diseño del trabajo, recolección de datos, redacción del manuscrito. Natasha Smiliansky: concepto y diseño del trabajo, recolección de datos, redacción del manuscrito.

Andrea Vaucher: concepto y diseño del trabajo, recolección de datos, revisión crítica del manuscrito. 